2004

\title{
Is the Fusiform Face Area Specialized for Faces, Individuation, or Expert Individuation?
}

Gillian Rhodes

Graham Byatt

Patricia T. Michie

Aina Puce

Follow this and additional works at: https://researchrepository.wvu.edu/faculty_publications

\section{Digital Commons Citation}

Rhodes, Gillian; Byatt, Graham; Michie, Patricia T.; and Puce, Aina, "Is the Fusiform Face Area Specialized for Faces, Individuation, or Expert Individuation?" (2004). Faculty Scholarship. 204.

https://researchrepository.wvu.edu/faculty_publications/204 


\title{
Is the Fusiform Face Area Specialized for Faces, Individuation, or Expert Individuation?
}

\author{
Gillian Rhodes $^{1}$, Graham Byatt ${ }^{1}$, Patricia T. Michie ${ }^{1,2}$, \\ and Aina Puce P,4,5 $^{3,5}$
}

\begin{abstract}
Several brain imaging studies have identified a region of fusiform gyrus (FG) that responds more strongly to faces than common objects. The precise functional role of this fusiform face area (FFA) is, however, a matter of dispute. We sought to distinguish among three hypotheses concerning FFA function: face specificity, individuation, and expert individuation. According to the face-specificity hypothesis, the FFA is specialized for face processing. Alternatively, the FFA may be specialized for individuating visually similar items within a category (the individuation hypothesis) or for individuating within categories with which a person has expertise (the expert-individuation hypothesis). Our results from two experiments supported the face-specificity hypothesis. Greater FFA activation to faces than Lepidoptera, another homogeneous object class, occurred during both free viewing and individuation, with similar FFA
\end{abstract}

\section{INTRODUCTION}

I never forget a face, but in your case I'll make an exception.

\section{- Groucho Marx}

We cannot, of course, choose to forget a face. In fact, we can remember hundreds or even thousands of faces, despite their similarity as visual patterns (Bahrick, Bahrick, \& Wittlinger, 1975). To do so, we must be able to individuate (discriminate) many faces. This individuation cannot be done using simple feature cues because all faces have the same basic features in the same common configuration (Diamond \& Carey, 1986). Even when an additional feature, such as a beard, is present, it will not uniquely differentiate that face from other bearded faces. Instead, faces appear to be individuated using subtle configural cues about the spatial relations between features (Murray, Yong, \& Rhodes, 2000; Searcy \& Bartlett, 1996; Bartlett \& Searcy, 1993; Rhodes, Brake, \& Atkinson, 1993; for reviews, see Peterson \& Rhodes, 2003) or between the facial configuration and a prototypical or norm face (Leopold, O' Toole, Vetter, \& Blanz, 2001; Rhodes, 1996; Diamond \& Carey, 1986). In con-

\footnotetext{
${ }^{1}$ University of Western Australia, ${ }^{2}$ University of Newcastle Australia, ${ }^{3}$ Swinburne University of Technology, ${ }^{4}$ Austin \& Repatriation Medical Centre, ${ }^{5}$ West Virginia University
}

activation to Lepidoptera and common objects (Experiment 1). Furthermore, during individuation of Lepidoptera, $83 \%$ of activated FG voxels were outside the face FG region and only $15 \%$ of face FG voxels were activated. This pattern of results suggests that distinct areas may individuate faces and Lepidoptera. In Experiment 2, we tested Lepidoptera experts using the same experimental design. Again, the results supported the face-specificity hypothesis. Activation to faces in the FFA was greater than to both Lepidoptera and objects with little overlap between FG areas activated by faces and Lepidoptera. Our results suggest that distinct populations of neurons in human FG may be tuned to the features needed to individuate the members of different object classes, as has been reported in monkey inferotemporal cortex, and that the FFA contains neurons tuned for individuating faces.

trast, objects may be recognized (at least at the basic level, Rosch, Mervis, Gray, Johnson, \& Boyes-Braem, 1976) using their parts and categorical spatial relations between those parts (Biederman, 1987).

Several lines of evidence suggest that this ability to individuate faces may result from the operation of a specialized neural module that encodes faces, and not other objects. First, electrophysiological studies have identified neurons in the temporal cortex of monkeys that respond selectively to faces and not to other stimulus categories (Desimone, 1991; Harries \& Perrett, 1991). Second, lesions of the occipito-temporal cortex in humans can produce prosopagnosia, an inability to recognize familiar faces (De Renzi, Perani, Carlesimo, Silveri, \& Fazio, 1994; Damasio, Damasio, \& Van Hoesen, 1982), which can occur independently of deficits in object recognition (Farah, Wilson, Drain, \& Tanaka, 1995; De Renzi, Faglioni, Grossi, \& Nichelli, 1991, reviewed by Farah, 1996). Deficits in object recognition can also occur independently of deficits in face recognition (Moscovitch, Winocur, \& Behrmann, 1997; McCarthy \& Warrington, 1986). This double dissociation suggests that the neural systems for recognizing faces and objects may be distinct. Third, electrical recordings from the brain surface of epilepsy patients have shown a set of face-specific event-related potentials in bilateral posterior fusiform gyrus (FG) occurring within a second 
of face onset (Allison, Ginter, et al., 1994; Allison, McCarthy, Nobre, Puce, \& Belger, 1994; Allison, Puce, Spencer, \& McCarthy, 1999). Fourth, electrical stimulation of these face-specific sites can produce a transient inability to identify previously familiar faces (Puce, Allison, \& McCarthy, 1999; Allison, Ginter, et al., 1994). Fifth, functional neuroimaging studies in normal humans consistently show activation of the FG to faces (Haxby, Hoffman, \& Gobbini, 2000; Haxby et al., 2001; Kanwisher, McDermott, \& Chun, 1997; McCarthy, Puce, Gore, \& Allison, 1997; Puce, Allison, Gore, \& McCarthy, 1995; Puce, Allison, Asgari, Gore, \& McCarthy, 1996; Sergent, Ohta, \& MacDonald, 1992) and this area appears to play a role in individuating faces, not just detecting them (Gauthier, Tarr, Moylan, Skudlarski, et al., 2000; George et al., 1999; see Haxby et al., 2000, for a review). In contrast, neighboring areas (in ventral occipito-temporal cortex, especially in the left hemisphere) respond selectively to objects (Allison et al., 1999; McCarthy et al., 1997; Kanwisher, Chun, McDermott, \& Hamilton, 1996; Malach et al., 1995). ${ }^{1}$ Sixth, newborns look longer at faces than at other stimuli with similar low-level properties (Johnson, Dziurawiec, Ellis, \& Morton, 1991) and rapidly learn to discriminate familiar from unfamiliar faces (Bushnell, 2001; Walton \& Bower, 1993). Furthermore, the first 6 months of life appears to be a critical period for developing the ability to code configural information in faces (Le Grand, Mondloch, Maurer, \& Brent, 2001). These data in infants suggest that specialized innate mechanisms may underlie our ability to individuate faces.

Despite the evidence that human FG responds to faces, its precise role in face processing still remains controversial. One view is that $\mathrm{FG}$ is the site of a specialized neural module, the fusiform face area (FFA), which individuates faces, but not other objects (Kanwisher et al., 1997; McCarthy et al., 1997). We will refer to this as the face-specificity hypothesis. This modular view is motivated by the neurophysiological, neuropsychological, and developmental evidence reviewed above.

Another view is that the FFA plays a role whenever the members of a homogeneous class are individuated (Tarr \& Cheng, 2003; Gauthier, Anderson, Tarr, Skudlarski, \& Gore, 1997; Gauthier, Tarr, Moylan, Anderson, et al., 2000). A homogeneous class is one whose members have the same basic parts in the same spatial arrangement. Hence, exemplars cannot be individuated by their parts or other isolated feature cues, and must be individuated using more configural information.

From this viewpoint, FFA is involved in encoding the configural cues needed to individuate exemplars that share a spatial configuration. We will refer to this as the individuation hypothesis. This hypothesis gains plausibility from evidence that a region of FG identified as face-sensitive in previous studies is more active when homogeneous objects are discriminated at the subordinate (e.g., eagle, retriever) than basic (e.g., bird, dog) level (Gauthier et al., 1997). The same pattern of results also occurs when the FFA is identified individually for each participant in the study (Gauthier, Tarr, Moylan, Anderson, et al., 2000). In the absence of a direct comparison with individuation of faces, however, it remains possible that the FFA responds more strongly to faces than other homogeneous stimuli, and may therefore show face specificity (using the same logic used to identify the FFA, namely, greater activation to faces than other stimulus classes). A stronger case could be made for face specificity, however, if distinct areas could be found for faces and other homogeneous classes.

Kanwisher et al. (1997) favor the face specificity over the individuation hypothesis, on the grounds that the FFA is more active during individuation of faces than hands. Because faces are automatically individuated (Tanaka, 2001; Kanwisher et al., 1997; Fodor, 1983), whereas objects are not (Rosch et al., 1976), they used a one-back matching task to encourage subjects to individuate both categories of stimuli. However, it is not clear that this ensures equivalent levels of individuation for faces and hands. The problem is that although a detailed visual representation of each hand is needed to support "same" responses, simple feature cues may suffice for "different" responses. This would mean that FFA activity is stronger for faces than hands because faces are individuated automatically on all trials (irrespective of the task), whereas hands are only fully individuated on "same" trials (which make up a relatively small proportion of trials when random sequences of hands are used).

McCarthy et al. (1997) also favor the face-specificity hypothesis. They argued against the individuation hypothesis on the grounds that FG responds to faces, but not flowers, when presented against a baseline of nonface objects. Gauthier, Tarr, Moylan, Anderson, et al. (2000) have criticized the choice of flowers as a comparison stimulus, arguing that these have distinctive and unique features and do not, therefore, constitute a homogeneous class.

Another possibility is that the FFA plays a role in expert individuation (Haxby et al., 2000; Tarr \& Gauthier, 2000; Gauthier, Tarr, Anderson, Skudlarski, \& Gore, 1999; Gauthier, Skudlarski, Gore, \& Anderson, 2000). This modification of the individuation hypothesis is motivated by behavioral evidence that only experts encode the configural cues needed to individuate homogeneous exemplars (Gauthier \& Tarr, 1997; Diamond \& Carey, 1986). We will refer to this as the expertindividuation hypothesis. To test this hypothesis, Gauthier et al. (1999) trained people to categorize novel homogeneous objects (greebles) until they could be individuated as quickly as they could be categorized at a more general "family" level. Expertise-related processing was measured as the difference in FFA activity between upright and inverted greebles in a sequential matching task. Changes in expertise-related processing 
of greebles during training were examined by scanning five participants before, during, and after acquisition of greeble expertise. Throughout training, faces produced more activity than greebles in face-sensitive areas, but by the end of training, this difference was no longer significant in the face areas (middle and anterior FG regions in right and left hemispheres). These results are interpreted as support for the expert-individuation hypothesis. There are, however, some problems with the study, which weaken this conclusion. First, the face-sensitive regions were sampled as $8 \times 8$ voxel (each voxel $1.3 \times 1.7 \mathrm{~mm}$ ) areas centered on activation peaks to faces in middle and anterior FG in each hemisphere. These regions will not be exactly coextensive with face-sensitive cortex. Second, activation was not assessed directly using percent change in the fMRI signal, but by summing $t$ values generated from $t$ tests comparing upright and inverted sequential matching performance. It is not clear whether summed $t$ values are a good measure of activation, as $t$ values depend on the size of both signal (variance between upright and inverted conditions) and noise (variance within upright and inverted conditions). This is a particular problem if the conditions (faces, greebles) are tested in different imaging runs, as was the case here. Third, only a small sample of experts $(n=5)$ was tested. Finally, and perhaps most importantly, greebles have a very facelike structure, and may recruit FFA activity simply by virtue of their similarity to faces (see Kanwisher, 2000). A stronger test of the expertindividuation hypothesis may therefore require the use of less facelike homogeneous classes.

Another study by Gauthier et al. examined expert identification of cars and birds, which do not have a facelike structure (although birds certainly have faces). They found that activity in the right hemisphere FFA correlated with a behavioral measure of expertise for both car and bird experts, suggesting a role for this face-sensitive region in expert individuation. However, this conclusion is weakened by the fact that the correlation was only found for a location-matching task (where individuation was incidental) and not for an identification-matching task. Furthermore, although activity was greater to the expert than nonexpert object category in the center of this right hemisphere FFA (collapsing across task), faces generally activated the FFAs more strongly than expert object individuation. Finally, Kanwisher (2000) notes that when the FFA is defined in the conventional way, face activity in these subjects remains at least double activity for cars and birds viewed by experts.

These imaging studies suggest that the FFA, or regions close to it, are activated when experts individuate homogeneous objects with which they have expertise (greebles, birds, cars), but that these levels are lower than for faces, unless facelike objects are used (see Kanwisher, 2000). This pattern could be interpreted as evi- dence that the FFA contributes to expert individuation, when individuated objects show a degree of similarity to faces. Nevertheless, the FFA shows some face-specificity and so should not be characterized as an expert-individuation area. Alternatively, one might argue that the greater response to faces simply reflects the greater expertise that people have with faces, and that the FFA is well characterized as an expert-individuation area. This interpretation gains support from recent evidence that the FFA response to faces itself depends on expertise participants have with the population of faces viewed, showing higher activation to own-race than other-race faces (Golby, Gabrielli, Chiao, \& Eberhardt, 2001). However, this result does not provide strong evidence against face specificity because we would expect a face-specific FFA to respond more strongly to stimuli that resembled a face prototype derived from experience than to those that do not. On this view, other-race faces would activate the FFA less than ownrace faces because they are less like the (own-race) face prototype.

\section{Present Study}

The goal of this study was to distinguish among facespecificity, individuation, and expert-individuation hypotheses. We did this by performing two experiments using faces and Lepidoptera (butterflies and moths), and we functionally localized the FFA in each individual subject. Our analyses of Experiments 1 and 2 specifically included examining the behavior of the a priori defined FFA voxels, in addition to that of other activated voxels in the FG outside the FFA.

Experiment 1 was designed to discriminate between face-specificity and individuation hypotheses, by observing fMRI activation in face-selective regions of FG when viewing images of faces and Lepidoptera, a homogeneous object class whose members are not at all facelike. Common objects were also included for comparison. Activation was measured in two groups of subjects who were nonexperts (novices) with respect to Lepidoptera: a passive viewing group and an active individuation group trained prior to the fMRI session on half the images and asked to make old/new judgments during fMRI acquisition. These two groups will be referred to as untrained novices and trained novices, respectively. After completing the passive viewing imaging session, untrained novices completed another session in which they viewed the images again and were asked to identify which ones they had seen in the previous session, thus providing a second individuation test.

We first functionally identified FFA regions-of-interest (FFA ROIs) individually for each subject using the conventional method of finding FG voxels that are more active during passive viewing of faces than common objects (cf. Kanwisher et al., 1997) in an initial localizer task. We compared activation, as defined by percent 
fMRI signal change (\%MR signal) relative to fixation, within these FFA ROIs to the three types of stimuli (faces, Lepidoptera, and common objects) for each group/task. The face-specificity hypothesis predicts greater activation for individuation of faces than Lepidoptera, irrespective of task (a main effect of stimulus type). The individuation hypothesis predicts similar activation whenever individuation is required, that is, for Lepidoptera during individuation tasks, and for faces, during passive viewing as well as individuation because faces are automatically individuated irrespective of task (Tanaka, 2001; Kanwisher et al., 1997; Fodor, 1983) (an interaction between stimulus type and task).

As an additional analysis, we also examined whether distinct areas of FG (not limited to the FFA ROI) were activated by individuation of faces and Lepidoptera. The face-specificity hypothesis predicts that distinct areas will respond to faces and Lepidoptera, whether or not individuation is required (i.e., during passive viewing and individuation). In contrast, the individuation hypothesis predicts that the same FG regions will respond whenever individuation is required, irrespective of stimulus type, that is, for Lepidoptera during the individuation task and for faces in both tasks.

To foreshadow the results of Experiment 1, the FFA was more active during individuation of faces than Lepidoptera, indicating little support for the individuation hypothesis. Although predicted by the face-specificity hypothesis, this effect could also be due to differences in expertise, and may, therefore, be consistent with the expert-individuation hypothesis. Hence, we performed Experiment 2 to distinguish between the face-specificity and expert-individuation hypotheses by testing Lepidoptera experts in the passive viewing procedure of Experiment $1 .^{2}$ As for the untrained novices, the experts also completed a second session in which they were asked to identify which images they had been shown previously. The face-specificity hypothesis predicts that the FFA should respond more strongly to faces than Lepidoptera, even for the experts in both passive viewing and individuation conditions. It also predicts that distinct regions will respond to faces and Lepidoptera. In contrast, the expert-individuation hypothesis predicts that the FFA should be strongly activated by Lepidoptera in the experts and that the same region(s) will respond to faces and Lepidoptera.

\section{RESULTS}

\section{Experiment 1}

Here, we contrasted the "face-specificity" hypothesis with the "individuation" hypothesis. The former predicts greater FFA activation to faces than Lepidoptera in both the passive viewing and individuation groups. The latter predicts greater FFA activation to faces than Lepidoptera in the passive viewing condition (where faces, but not Lepidoptera, are individuated), but not in the individuation condition.

\section{Localizer Analysis}

All participants activated regions within the left and right FG more strongly to faces than to objects in the localizer task (Kolmogorov-Smirnov [KS] test, $z>3.08, p<.001$, uncorrected), with the activated volume being larger in the right $\left(1140 \mathrm{~mm}^{3}, S D=474 \mathrm{~mm}^{3}\right)$ than the left hemisphere $\left(576 \mathrm{~mm}^{3}, S D=402 \mathrm{~mm}^{3}\right), t(16)=6.1, p<.001$. These activated regions defined the FFA ROIs for each participant for the data analysis in the main task.

\section{FFA ROI Analysis (Main Task)}

Analysis of the mean \%MR signal change relative to fixation as a function of viewing condition (passive viewing for untrained novices vs. individuation for trained novices) revealed that the level of activation in left and right hemisphere FFA ROIs was substantially affected by stimulus category, $F(2,30)=87.11, p<.001$, an effect that was moderated by interactions with viewing condition, $F(2,30)=5.35, p<.017$, and hemisphere, $F(2,30)=3.64, p<.05 .^{3}$ Overall, the FFA was significantly more activated by faces than Lepidoptera and common objects ( $p s<.001$; see Figure 1) in both hemispheres. Exploration of the interaction between stimulus category and viewing condition using Bonferroni-corrected multiple comparisons of simple viewing condition effects $(p<.05)$ demonstrated that individuation resulted in significantly increased \%MR signal in the FFA to Lepidoptera stimuli compared to passive viewing. No other contrast was significant. Comparisons of stimulus category within each viewing condition, again using Bonferroni adjustment, confirmed that in both conditions, faces resulted in significantly greater FFA activation than either Lepidoptera or objects, which did not differ.

For the untrained novices, we reexamined the effect of viewing condition (passive viewing vs. individuation) in a repeated measures analysis, by comparing their performance in Sessions 1 (passive viewing) and 2 (individuation). There was a highly significant effect of session, $F(1,8)=39.81, p<.001$, with individuation increasing percent signal change by approximately $50 \%$ (from $0.77 \%$ in Session 1 to $1.11 \%$ in Session 2: see Figure 1). There was a significant main effect of stimulus category, $F(2,16)=49.62, p<.0001$, as before, but no interaction between session and stimulus category (or session and hemisphere) and Figure 1 shows that the increase in \%MR signal for individuation compared with passive viewing was not restricted to Lepidoptera. Finally, irrespective of whether subjects were passively viewing the stimuli or attempting to identify previously seen stimuli, faces resulted in significantly higher signal change than either objects or Lepidoptera $(p<.001$ in both cases). 


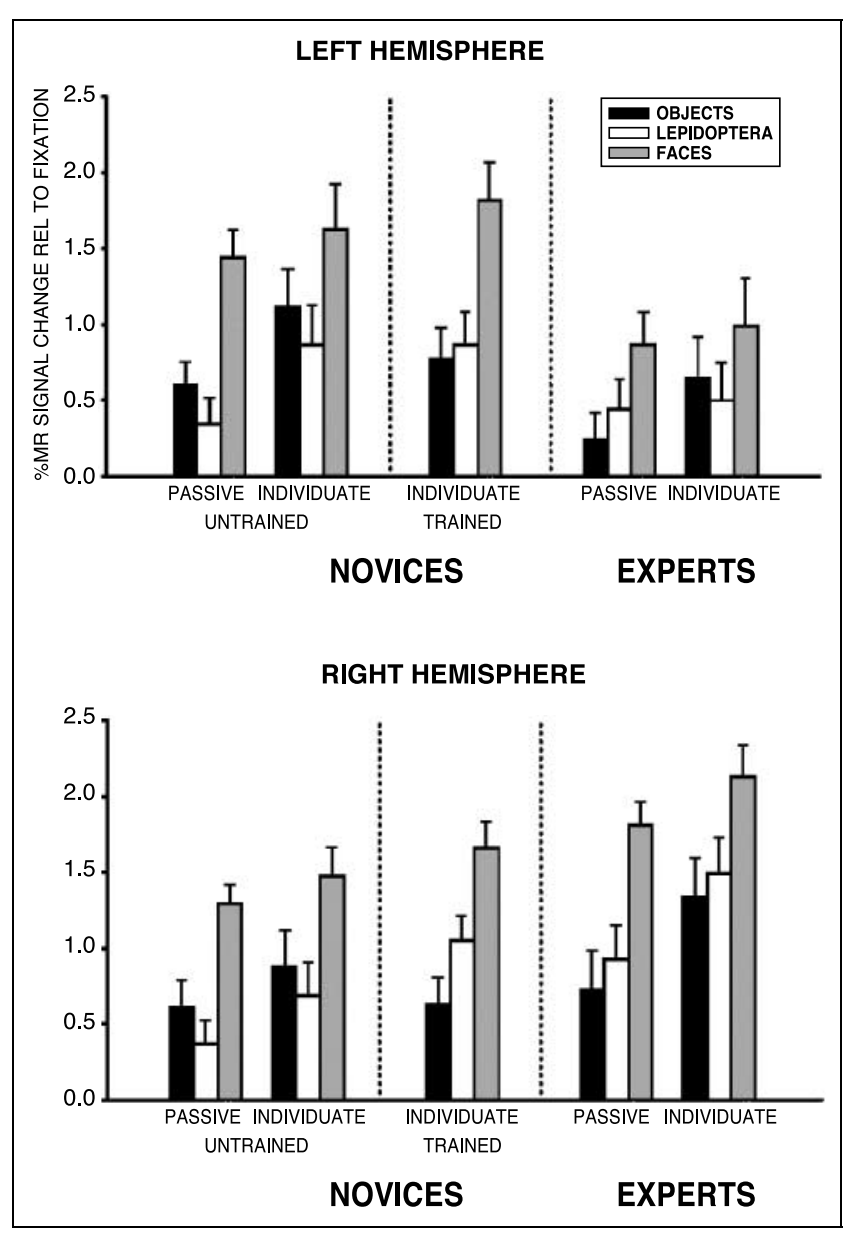

Figure 1. \%MR signal change (relative to fixation) within the functionally defined FFA ROI for each stimulus category for untrained novices, in the passive viewing and individuation conditions (Experiment 1), for trained novices in the individuation condition (Experiment 1), and for experts in passive viewing and individuation conditions (Experiment 2). Error bars denote standard errors of the mean.

Therefore, although individuation of Lepidoptera by both trained and untrained novices increased activation in FFA ROIs relative to passive viewing by untrained novices, the degree of activation still did not approach that generated by faces. Furthermore, the increased FFA activation for individuation (Session 2) over passive viewing (Session 1) in untrained novices was not specific to Lepidoptera. Overall, these results do not support the individuation hypothesis, which predicts similar activation for faces and Lepidoptera during individuation, and are more consistent with the facespecificity hypothesis. The possibility remains, however, that the difference in FFA activation to faces and Lepidoptera reflects differences in expertise. Although old/new identification of Lepidoptera (66.5\%) by participants in the individuation group was significantly above chance, $t(7)=4.09, p<.005$, it was substantially poorer than for faces $(90.5 \% ; p<.005$, Bonferroni correction) and objects (92.4\%; $p<.005$, Bonferroni correction), $F(2,14)=28.81, p<.001$, despite the extensive practice with Lepidoptera. ${ }^{4}$ In Experiment 2, we will explore the contribution of expertise to activation of the FFA by Lepidoptera, by examining activation for Lepidoptera experts.

\section{FG Regional Overlap Analysis}

Here we examined the degree of overlap between FG regions activated by faces and Lepidoptera. The facespecificity hypothesis predicts that faces and Lepidoptera will activate distinct FG areas. In contrast, the individuation hypothesis predicts that the same FG areas will be activated whenever exemplars are individuated, that is, by both Lepidoptera and faces during individuation and by faces only during passive viewing. To obtain an index of the degree of activation overlap, the regions within FG that were activated by faces versus objects and Lepidoptera versus objects were determined and then the percentage of common voxels (relative to faces vs. objects) was calculated in passive viewing untrained novices and individuating trained novices (Table 1). The degree of overlap between the FG areas activated by faces and Lepidoptera was in general low (mean $10.3 \%)$, but substantially larger in the individuation (15.4\%) than in the passive viewing conditions $(5.7 \%)$, $F(1,15)=9.46, p<.008$, suggesting greater engagement of the FG areas that respond to faces by another set of homogeneous stimuli when individuation of those stimuli is required. A similar increase in the degree of overlap was evident in the untrained novices during Session 2 when they attempted to individuate: The overlap increased from $5.7 \%$ to $13.6 \%, F(1,8)=5.30, p<.050$, and was greater in the right hemisphere $(11.9 \%)$ than the left $(7.4 \%), F(1,8)=5.94, p<.041$. However, the degree of overlap in general was quite low.

As a check on the validity of this approach for determining the level of common activation within an area by two tasks, we also determined the degree of overlap between FG areas activated by the two sets of face stimuli (vs. objects) in the localizer task and the main task. Despite the differences in actual stimuli, epoch length, repetition of stimuli, interstimulus interval, and signal-tonoise ratios between the two-face (vs. objects) conditions, it was expected that coactivated areas would be larger for the two face tasks than for the faces and Lepidoptera. The expectation was confirmed: Using the same procedures, the overlap overall was approximately 2.5 times higher (26.2\%) for the two face tasks than for the faces versus Lepidoptera comparison (10.3\%), with no difference between the passive (27.2\%) and individuation (25.1\%) groups, or the left (26.2\%) and right (26.2\%) hemispheres (averaging across passive viewing and individuation groups). The difference between the level of overlap in regions activated by faces and Lepidoptera compared with the two face tasks was 
significant, $F(1,15)=18.90, p<.001$. While the degree of overall overlap of $26.2 \%$ in activated areas by the two face tasks seems modest, given the apparent robust consistency of previous research demonstrating activation of FG to faces, it should be noted that the measure of overlap used here is a conservative index as it is the degree of overlap in activated regions thresholded to the same conservative criterion for both tasks.

The modest degree to which the Lepidoptera activated FG regions sensitive to faces could be due to Lepidoptera activating only this particular area of FG and no other FG areas. This possibility was assessed by determining the percentage of the FG area activated by Lepidoptera (vs. objects) that did not overlap with the face (vs. objects) area within FG. On average, $83 \%$ of the FG area activated by Lepidoptera did not overlap with the face FG area. There was no significant difference between the passive (81\%) and individuation (85\%) groups or between the left (87\%) and right (75\%) hemispheres.

We also considered whether the modest degree of overlap could be due to small FG volumes activated by Lepidoptera (vs. objects). Similarly sized volumes were activated by faces and Lepidoptera in both the left and right FG areas for each of the viewing conditions except for Lepidoptera in passive viewing by untrained novices where the activated FG volume was approximately one-third of the size of face volume,
$F(1,8)=7.27, p<.024$ (Table 1 ). In all individuation conditions, similarly sized FG volumes were activated by faces and Lepidoptera ( $p>.05$ for all comparisons). However, individuation resulted in increased FG activation volumes to both faces and Lepidoptera: in the trained novices compared with the passive viewing untrained novices [group effect: $F(1,14)=21.35, p<.001$ ] and in individuating untrained novices [session effect: $F(1,8)=5.85, p<.042]$. Therefore, the magnitude of FG volumes activated by faces (vs. objects) and Lepidoptera (vs. objects) can account for variation between viewing conditions in the degree of overlap between faces and Lepidoptera. However, it cannot account for the relatively modest degree of overlap between faces and Lepidoptera compared with the two sets of face stimuli. Inspection of Table 1 indicates that trained novices had similar volumes activated by faces and Lepidoptera, but their face-Lepidoptera overlap (15.4\%) was substantially less than their face-face overlap (25.1\%).

\section{Experiment 2}

\section{Behavioral Assessment of Expertise}

Experts and nonexperts completed an old/new recognition task and a classification task with Lepidoptera (different from those seen in the scanner but from the

Table 1. Mean (SEM) Right and Left Hemisphere Activated FG Volumes (in $\mathrm{mm}^{3}$ ) for Face versus Object Contrast and Lepidoptera versus Object Contrast in Each Group and Condition

\begin{tabular}{|c|c|c|c|c|c|c|}
\hline \multirow[b]{2}{*}{ Group/Condition } & \multicolumn{3}{|c|}{ Faces versus Objects } & \multicolumn{3}{|c|}{ Lepidoptera versus Objects } \\
\hline & Left & Right & $M$ & Left & Right & $M$ \\
\hline \multicolumn{7}{|l|}{ Untrained Novices } \\
\hline Passive viewing & $868(224)$ & $1422(355)$ & $1145(265)$ & $356(98)$ & $334(124)$ & $345(100)$ \\
\hline Percent overlap & & & & $2.4(1.6)$ & $9.1(3.3)$ & $5.7(1.7)$ \\
\hline \multicolumn{7}{|l|}{ Untrained Novices } \\
\hline Individuation & $1529(281)$ & $1742(414)$ & $1636(414)$ & $1038(350)$ & $1223(513)$ & $1131(352)$ \\
\hline Percent overlap & & & & $12.4(4.0)$ & $14.8(3.1)$ & $13.6(3.3)$ \\
\hline \multicolumn{7}{|l|}{ Trained Novices } \\
\hline Individuation & $1096(178)$ & $2016(558)$ & $1556(439)$ & $1312(164)$ & $1856(258)$ & $1584(374)$ \\
\hline Percent overlap & & & & $17.1(3.7)$ & $13.8(4.1)$ & $15.4(2.3)$ \\
\hline \multicolumn{7}{|l|}{ Experts } \\
\hline Passive viewing & $1440(255)$ & $2032(375)$ & $1736(282)$ & $872(171)$ & $1040(332)$ & $956(243)$ \\
\hline Percent overlap & & & & $6.0(1.6)$ & $7.5(3.5)$ & $6.7(1.8)$ \\
\hline \multicolumn{7}{|l|}{ Experts } \\
\hline Individuation & $1664(626)$ & $2504(627)$ & $2084(439)$ & $1704(627)$ & $944(333)$ & $1324(374)$ \\
\hline Percent overlap & & & & $14.2(4.3)$ & $3.6(3.4)$ & $8.9(3.5)$ \\
\hline
\end{tabular}

Percent overlap between FG areas activated by Lepidoptera and faces is also shown. 
same source). In the old/new task, experts recognized more Lepidoptera $(M=71.6 \%, S D=11.8 \%)$ than nonexperts $(M=65.9 \%, S D=10.2 \%)$, but this difference was not significant, $F(1,15)=2.35, p>.05$. There was however, a significant Group $\times$ Gender interaction, $F(1,15)=9.28, p<.008$, attributable to superior performance of the male Lepidoptera experts (77.0\%) compared to male nonexperts $(56.3 \%, p<.011$, uncorrected). Experts correctly classified significantly more Lepidoptera $(M=73.4 \%, S D=7.8 \%)$ than nonexperts $(M=61.7 \%, S D=9.8 \%), F(1,15)=12.57, p<.003$. There were no gender differences on the classification task. Age did not predict performance on either task.

\section{Localizer Analysis}

All experts activated regions within the left and right FG more strongly to faces than objects in the localizer task (KS test, $z>3.08, p<.001$, uncorrected) with the activated volume being larger in the right $\left(912 \mathrm{~mm}^{3}\right.$, $\left.S D=566 \mathrm{~mm}^{3}\right)$ than the left hemisphere $\left(624 \mathrm{~mm}^{3}\right.$, $\left.S D=574 \mathrm{~mm}^{3}\right), t(7)=3.67, p<.008$. These activated regions defined FFA ROIs for each participant in the main task.

\section{FFA ROI Analysis (Main Task)}

Analysis of the \%MR signal change relative to fixation revealed a similar pattern to the novices. Activation of the FFA ROIs was substantially affected by stimulus category in both hemispheres, $F(2,14)=26.96, p<.001$ (see Figure 1). Multiple comparisons of stimulus categories revealed that even in the experts, activation generated by faces was significantly larger than to either Lepidoptera $(p<.001)$ or objects $(p<.001)$. Importantly, the latter did not differ significantly. These findings are consistent with the face-specificity hypothesis. There was also a significant hemisphere effect, $F(1,7)=6.63, p<.001$, and a Hemisphere $\times$ Category interaction, $F(2,14)=4.98$, $p<.047$, due to larger \%MR signal change in the right hemisphere overall and a markedly larger \%MR signal change to faces in the right hemisphere (see passive condition for experts in Figure 1).

In contrast with novices, activation of the FFA ROIs did not increase significantly when the experts attempted to individuate in the second session (see Figure 1). Main effects and interactions over the two sessions combined in experts were otherwise similar to those seen in passive viewing.

Performance by the experts on the classification task was related to right hemisphere \%MR signal change in FFA ROIs to Lepidoptera $(r=.80, p<.018)$ and objects $(r=.84, p<.010)$, but not faces $(r=-.06)$ during individuation only. Both of these correlations increased after controlling for gender $(r=.93, p<.002$, and $r=.95, p<.001)$. Lepidoptera and object activation were also highly correlated $(r=.98, p<.001)$, and neither correlation with classification performance was significant after adjusting for the other. Correlations between classification performance and left hemisphere activation did not approach significance and nor was there evidence of similar correlations under passive viewing. No correlations with performance on the old/ new judgment task were significant. That is, behavioral performance on the task that distinguished between Lepidoptera experts and nonexperts overall, namely, the classification task, correlated highly with the strength of right hemisphere activation to Lepidoptera during individuation. However, as behavioral performance was also highly correlated with degree of right hemisphere activation to objects during individuation, the correlation with Lepidoptera activation seems unlikely to be due solely to expert individuation. These correlations could possibly reflect greater attention by experts during individuation. However, it is not clear why such an attentional effect would not also be found for faces or for left hemisphere individuation.

\section{FG Regional Overlap Analysis}

Analysis of the degree of overlap between the FG areas activated by faces and Lepidoptera during passive viewing was again small (6.7\%), and substantially less than the degree of overlap in activated FG areas in the two face tasks $(28.3 \%), F(1,7)=21.03, p<.003$. The overall degree of overlap between faces and Lepidoptera increased only modestly (from $6.7 \%$ to $8.9 \%$ ) and nonsignificantly from the first (passive viewing) to the second (individuation) session. There was, however, a significant Session $\times$ Hemisphere interaction, $F(1,7)=8.55, p<.022$, due to an increase in overlap in the left hemisphere in the second session (individuation) (from $6.0 \%$ to $14.2 \%$ ) and a reduction in the right (from $7.5 \%$ to $3.6 \%$ ). On average, $86 \%$ of the FG area activated by Lepidoptera did not overlap with the face FG area during passive viewing. This showed a small, but nonsignificant, increase to $90 \%$, when individuating, in the second session. Inspection of Table 1 shows that the smaller overlap between face and Lepidoptera voxels than between the two face tasks cannot be accounted for by differences in volumes of the Lepidoptera and face-activated voxels. The faceLepidoptera overlaps were much smaller (less than half) than expected based on differences in volume. Therefore, relatively distinct regions respond to faces and Lepidoptera even in Lepidoptera experts, consistent again with the face-specificity hypothesis.

\section{Lepidoptera Experts versus Untrained Novices}

As identical viewing conditions were used for the experts in Experiment 2 and untrained novices in Experiment 1, direct comparisons of the magnitude of \%MR signal 
change in FFA ROIs to faces and Lepidoptera, and of regional overlap in the two groups, were possible.

\section{FFA ROI Analysis (Main Task)}

Lepidoptera experts overall showed larger \%MR signal change relative to fixation than novices in the right hemisphere in both passive viewing and individuation [Group $\times$ Hemisphere: $F(1,14)=5.73, p<.030$, and $F(1,14)=8.92, p<.009$, respectively]. However, in passive viewing (but not individuation), there was evidence of differential activation across the stimulus categories in the two groups [Category $\times$ Group: passive: $F(2,30)=5.93, p<.007$; individuation: $F(2,30)=0.55, p>.05]$, which varied with hemisphere [Group $\times$ Category $\times$ Hemisphere: Passive: $F(2,30)=$ 7.11, $p<.007$; individuation: $F(2,30)=0.64, p>.05$ ]. Both of these passive viewing interactions moderated a very substantial main effect of category [passive: $F(2,30)=93.84, p<.001$; individuation: $F(2,30)=17.55$, $p<.001]$. Multiple comparisons of stimulus categories with Bonferroni correction $(p<.05)$ in the passive viewing condition revealed that in both experts and the untrained novices, left and right hemisphere activation to faces was significantly larger than to either Lepidoptera or objects, with no significant difference between the latter two categories, findings consistent with the face-specificity hypothesis. However, as Figure 1 suggests, Lepidoptera activation in the right hemisphere was larger in experts than untrained novices, although this difference was only significant using uncorrected $p$ values $(p<.020)$ and furthermore, there was a similar difference for faces $(p<.011$, uncorrected). There was greater signal change in the right hemisphere of the experts to all categories of stimuli and viewing conditions (see Figure 1).

To assess the expert-individuation hypothesis more specifically, we conducted a separate comparison of Lepidoptera and object \%MR signal change in FFA ROIs in the untrained novices and experts in the passive viewing condition only. While neither category of stimulus nor group were significant, there was a substantial interaction between the two, $F(1,15)=12.48, p<.003$. This interaction was evident in both the left and right hemisphere activation patterns when analyzed separately, $F(1,15)=11.69, p<.004$, and $F(1,15)=7.71$, $p<.014$. In both hemispheres, \%MR signal change was larger for Lepidoptera than objects in experts ( $0.68 \%$ vs. $0.49 \%)$, whereas the opposite pattern was evident in untrained novices ( $0.36 \%$ vs. $0.61 \%)$. However, simple effect comparisons of Lepidoptera versus objects were significant for untrained novices $(p<.009$, uncorrected) and not for experts $(p=.106$, uncorrected). Note that experts also had similar \%MR signal change for Lepidoptera and objects during individuation (Figure 1). Furthermore, even in experts, \%MR signal change in FFA ROIs to faces (1.34\%) was double that to Lepidoptera. Finally, there was no interaction between group and stimulus category during individuation. These results are more consistent with the face-specificity hypothesis than the expert-individuation hypothesis.

\section{FG Regional Overlap Analysis}

The degree of overlap between FG areas activated by faces and Lepidoptera was not significantly larger overall in the Lepidoptera experts than the untrained novices, in either the passive viewing or individuation conditions. There was a trend for the experts to show greater overlap than novices in the left hemisphere $(10.1 \%$ vs. $7.4 \%)$, but the reverse pattern was evident in the right hemisphere (5.5\% vs. 12.0\%) [Hemisphere $\times$ Group interaction: $F(1,14)=6.80, p<.020]$. In addition, individuation resulted in a substantial increase in left hemisphere overlap relative to passive viewing (from $4.2 \%$ to $13.2 \%$ ) [Session $\times$ Hemisphere interaction: $F(1,15)=4.41, p<.053]$, a pattern evident in both untrained novices and experts.

Overall, experts activated larger FG volumes to both faces (vs. objects) and Lepidoptera (vs. objects) compared with untrained novices under passive viewing conditions, $F(1,14)=15.00, p<.002$ ) (see Table 1 ) and the FG volume activated to faces was larger than to Lepidoptera, $F(1,14)=9.43, p<.008$, a pattern that did not vary across the two groups, $F(1,15)=0.002, p>.05$. That is, the extent of FG activation to Lepidoptera was larger in experts than untrained novices, but this effect was not restricted to Lepidoptera activation: When passively viewing stimuli, experts activated larger FG volumes to both categories of stimuli, faces, and Lepidoptera. Hence, it seems unlikely that the increased volume of activation to Lepidoptera was the result solely of expertise. In contrast, individuation resulted in a substantial increase in the volume of activated FG tissue in the novices, so much so, that the overall group difference was no longer significant, $F(1,15)=3.00$, $p<.104$. In general, however, FG volumes activated by experts were still larger than in untrained novices (see Table 1), with the exception of Lepidoptera activation in the right hemisphere [Category $\times$ Hemisphere $\times$ Group interaction: $F(1,15)=4.55, p<.050$ ].

\section{Location of Lepidoptera and Face Activation}

Figure 2 illustrates the location of activation to Lepidoptera (vs. objects) and faces (vs. objects) during passive viewing by untrained novices and experts. As a group, the novices showed little Lepidoptera activation (beyond that obtained for objects). Individual novices did show small volumes of cortex that responded to Lepidoptera (see Table 1), but these do not appear to be located consistently enough to emerge in this group analysis. Experts, who individually had larger volumes of 


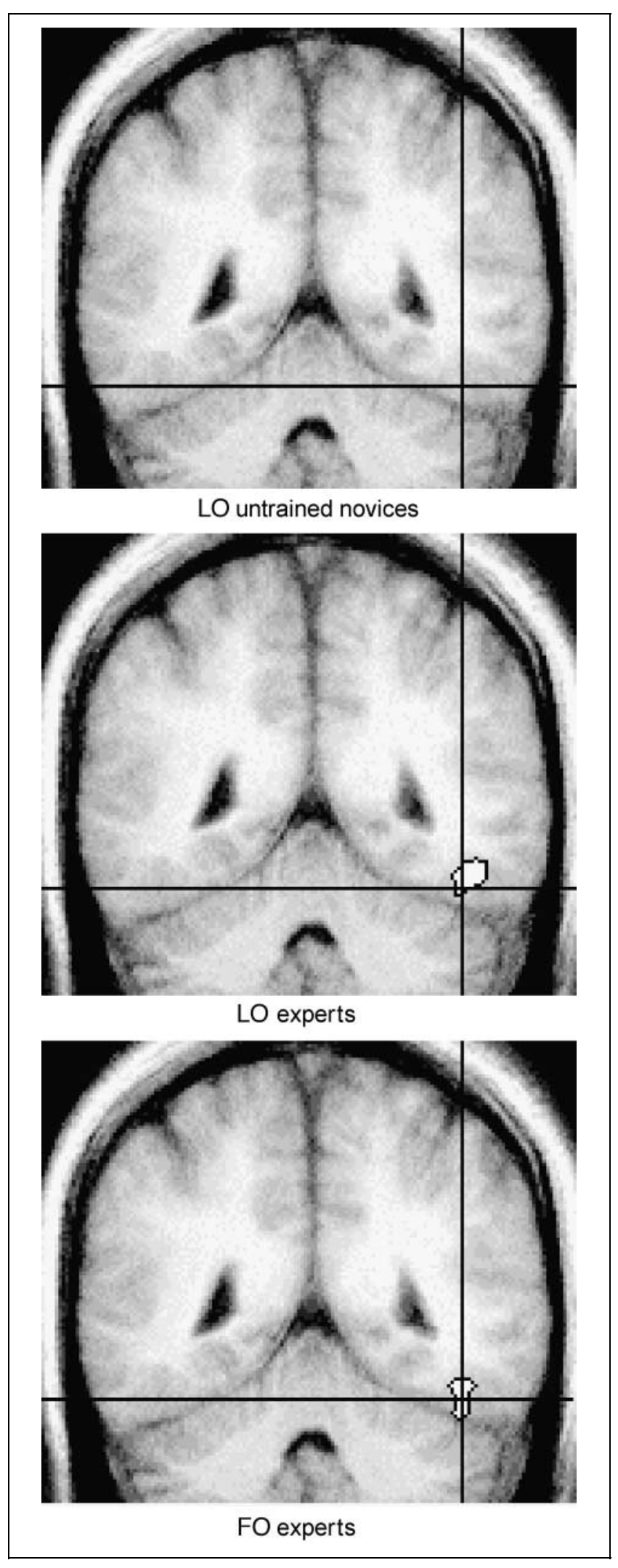

Figure 2. Coronal slices showing the location of activation foci to the Lepidoptera-object contrast in untrained novices (top) and experts (middle), during passive viewing. The face-object contrast for experts is also shown (bottom). The crosshairs indicate the center of the face-object activation.
Lepidoptera-activated cortex than untrained novices (Table 1), showed clear Lepidoptera activation located in mid-fusiform cortex. Inspection of Figure 2 shows that Lepidoptera activation overlapped with face activation, extending laterally (and posteriorly, not shown) beyond it. Face activation extended ventrally below the Lepidoptera activation. The figure shows clear overlap between the two areas, but it is important to note that for individual experts (see overlap analysis), the amount of overlap was smaller than shown here for face and Lepidoptera voxels that were common to all the experts.

\section{DISCUSSION}

There is considerable evidence that a region of the FG, the FFA, plays an important role in face processing, but the precise nature of this role remains controversial. We have identified three hypotheses regarding the role of the FFA in face and object processing. The face-specificity hypothesis holds that the FFA is specialized for processing faces but not other objects. The individuation hypothesis holds that the FFA is recruited whenever individuation of members of a homogeneous class is required. The expert-individuation hypothesis holds that expertise is required to be able to make the fine distinctions necessary to individuate between highly homogeneous stimuli and therefore the FFA is recruited only for individuation of members of a homogeneous class with which we have expertise.

To distinguish between these three hypotheses, we measured fMRI activation in three groups of participants: untrained novices, trained novices, and experts, during passive viewing and individuation of faces, common objects, and Lepidoptera. Our design overcame some of the limitations of previous studies, allowing direct comparison of faces and members of another homogeneous class that are not facelike. Discrimination among species of Lepidoptera involves similar computational demands to the identification of faces. That is, both stimulus types share the same parts in approximately the same spatial configuration and are distinguished by subtle metric differences and pattern variation.

In Experiment 1, we contrasted the face-specificity hypothesis with the individuation hypothesis by comparing activation during passive viewing and active individuation in novices. The results supported the face-specificity and not the individuation hypothesis. We found greater FFA activity to faces than Lepidoptera and objects irrespective of viewing condition with no difference between Lepidoptera and objects. Additional support for the face-specificity hypothesis comes from our finding that only $15.4 \%$ of the FG voxels engaged in individuation of faces were also active when individuating Lepidoptera. Approximately $83 \%$ of the FG voxels activated by Lepidoptera were outside the FG face area. Thus, even when subjects are actively individuating Lepidoptera and faces, the FFA response is significantly 
greater to faces and distinct regions of the FG are involved. These results support the face-specificity hypothesis over the individuation hypothesis but leave open the possibility that expertise with a stimulus class is a prerequisite for FFA involvement.

In Experiment 2, we contrasted the face-specificity hypothesis with the expert-individuation hypothesis by comparing activation between the three stimulus classes in Lepidoptera experts. Again, the results supported the face-specificity hypothesis. Activation to faces in the FFA was greater than to Lepidoptera and objects with little overlap between FG areas activated by faces and Lepidoptera. Approximately $86 \%$ of FG voxels activated by Lepidoptera were outside the FG face area.

Gauthier et al. (1997) and Gauthier, Tarr, Moylan, Anderson, et al. (2000) found greater FFA activity for subordinate- versus basic-level identification and have suggested that the FFA is recruited whenever members of an homogeneous class are individuated. Similarly, we found that FFA activation to Lepidoptera was greater during individuation (trained novices) than passive viewing (untrained novices). However, when we compared individuation and passive viewing in the same participants (untrained novices), we found a similar increase in FFA activation to objects during individuation, which suggests that the greater Lepidoptera activation may not reflect an FFA contribution to within-class individuation (Experiment 1). In addition, we still observed significantly greater activation for faces than Lepidoptera in both viewing conditions. Furthermore, although the degree of overlap between areas activated by faces and Lepidoptera was larger in the individuation than in the passive viewing group (15.4\% compared to 5.7\%), most of the FG voxels active to Lepidoptera were still outside of the FG face area (85\%, Experiment 1).

In another study, Gauthier, Skudlarski, et al. (2000) found that FFA activity correlated with a behavioral measure of expertise (car and bird experts). However, faces generally produced greater FFA activity than cars and birds viewed by experts. In the present study, a behavioral measure of expertise (from the classification task) also correlated with right hemisphere FFA activation to Lepidoptera in Lepidoptera experts during individuation but this finding cannot be unambiguously attributed to an effect of expert individuation since the same behavioral measure also correlated with right hemisphere FFA activation to objects. Finally, as for the novices, FFA activation in Lepidoptera experts was substantially and significantly greater for faces than Lepidoptera and the majority of FG voxels activated to Lepidoptera were outside the face FG area (over 86\%).

A group analysis confirmed that the areas of midfusiform cortex activated by Lepidoptera and faces in experts were not coextensive (Figure 2). This result corroborates the overlap analysis, which showed little common activation by Lepidoptera and faces in individual experts, and suggests that expertise with specific classes of objects recruits distinct regions of fusiform cortex. Individual novices had small volumes of Lepidoptera-activated cortex (Table 1), but these were apparently not consistently located enough for any common Lepidoptera-activated area to emerge in the group analysis.

Some researchers suggest that face processing predominantly activates the right hemisphere (Kanwisher et al., 1997; McCarthy et al., 1997) whereas others hold that face processing involves both left and right FG (Gauthier et al., 1999; Haxby et al., 1999) although each may contribute in a different way (e.g., Rossion, Dricot, Devolder, Bodart, \& Crommelinck, 2000). We found that all participants showed regions in both left and right FG that activated more strongly to faces than to objects in the localizer task. However, the total volume of activation was significantly larger in the right than the left hemisphere ROIs. Nevertheless, within these regions, the pattern of differential responses to each of the stimulus classes was generally equivalent across hemisphere for all subject groups.

The particular Lepidoptera used in the study were not known to the experts, so it is unlikely that they would have used their names. Even if they had, object naming does not normally activate the FG (e.g., Price, Moore, Humphreys, Frackowiak, \& Friston, 1996). Therefore, the FG activation to Lepidoptera seen in experts is unlikely to reflect naming. Attentional effects may contribute to the differences in Lepidoptera activation by experts and novices seen here (cf., Wojciulik, Kanwisher, \& Driver, 1998). Such effects may be an inevitable component of expert processing, given the great interest that experts have in their objects of interest. A challenge for future research is to isolate the components of expert processing due to greater interest and those due to greater visual expertise per se.

In terms of our stated hypotheses, the present results clearly support the face-specificity hypothesis. Our conclusion is not only based on the finding of greater activation to faces than other stimuli in the FFA, but also on the fact that different regions of the FG are recruited for face and Lepidoptera processing. The results suggest that the FG is the site of a neural module, the FFA, which is specialized for the processing of faces, but not other objects (as suggested by Kanwisher et al., 1997; McCarthy et al., 1997). This suggests that distinct populations of neurons in the FG, largely outside the FFA, are tuned to the features needed to discriminate different classes of objects. A similar organization has been observed in monkeys trained as experts on different object classes (Logothetis, 1998; Logothetis, Pauls, \& Poggio, 1995). Distinct populations of neurons may be recruited whenever qualitatively different kinds of features are needed for each class. These results may challenge the recent claim that a single system is used to recognize all objects (Tarr \& Cheng, 2003). Future research is needed to determine 
whether distinct computations are indeed performed in these distinct areas.

\section{METHODS}

\section{Experiment 1}

\section{Participants}

In Experiment 1, 17 adult Caucasian participants (age 23-50 years, $M=30.6, S D=8.6,11$ women) were tested. Nine untrained novices participated in the passive viewing condition $(M=28.5$ years, $S D=7.4$, 7 women) and eight trained novices participated in the individuation condition $(M=33.0$ years, $S D=9.7$, 4 women). All participants were healthy, had normal or corrected-to-normal vision, and were right-handed as assessed using the Edinburgh Handedness Inventory (Oldfield, 1971). None of the participants in Experiment 1 had expert knowledge with Lepidoptera and are referred to as novices (trained or untrained). In Experiments 1 and 2, all participants provided informed consent in a study approved by two institutional ethics committees (University of Western Australia and Swinburne University of Technology).

\section{Stimuli}

Localizer task. Digitized, gray-scale images of faces (10 men, 10 women; stimuli provided by N. Kanwisher) with varied facial expression in front to three-fourths profile views, common objects $(n=20)$, and a fixation stimulus consisting of a black cross on a white background were used in the localizer task.

Main (faces objects Lepidoptera) task. Digitized, grayscale images of 72 faces (36 men, 36 women) in front views with neutral expressions, 72 Lepidoptera viewed from above, with wings extended (36 butterflies, 36 moths selected from Lepidoptera reference books), and 72 common objects in canonical views (all stimuli were different from those used in the localizer task) were used in the experiment. A fixation stimulus consisted of a small black cross on a gray background. All stimuli were matched for overall luminance, contrast, and size.

\section{Procedure}

Images were rear projected onto a screen at the back of the magnet bore and viewed with a mirror mounted on the head coil. The faces, objects, and Lepidoptera in the main task had a viewing angle of approximately $11 \times 11^{\circ}$ (maximum horizontal and vertical extent on screen: $265 \times 265 \mathrm{~mm}$; viewing distance: $139.5 \mathrm{~cm}$ ). The faces and objects in the localizer task were approximately half this size.

Localizer task. Subjects were informed that they should simply attend to each image as it appeared and need not try to remember or make any judgments about the stimuli. There were two runs, each of $3 \mathrm{~min} 12 \mathrm{sec}$, consisting of 12 epochs of 20 images, with four epochs of each stimulus type: faces $(\mathrm{F})$, objects $(\mathrm{O})$, and fixation $(+)$. The order within Run 1 was $+\mathrm{FO}+\mathrm{OF}+\mathrm{FO}+\mathrm{OF}$, and for Run 2: $+\mathrm{OF}+\mathrm{FO}+\mathrm{OF}+\mathrm{FO}$. The localizer task was always presented first.

Images were presented for $300 \mathrm{msec}$ with a $500 \mathrm{msec}$ interstimulus interval consisting of a blank white screen (total of 16 sec/epoch). For each epoch, the (same) 20 images were presented in a random order. The TR of $2.1 \mathrm{sec}$ allowed a sampling of 7.6 volumes per epoch.

Main task (faces, objects, Lepidoptera). INDIVIDUATION GROUP (TRAINED NOVICES). Half the images from each stimulus category were studied prior to the scanning session. These study images were viewed for $15 \mathrm{~min}$ ( $5 \mathrm{~min}$ for each type), three times each day, for the 5 days prior to the imaging session. During the imaging session, subjects were presented with all the images and indicated which were, or were not, in the study set by pressing the left and right buttons of a button-box held on the stomach with the left and right thumbs, respectively. Responses were recorded to obtain performance measures for each subject. Different random sets of images were used as the study set for each subject.

PASSIVE VIEWING GROUP (UNTRAINED NOVICES). No images were studied prior to scanning. Scanning was divided into two functional imaging "sessions," which were separated by the acquisition of a high-resolution anatomical T1weighted imaging sequence. In Session 1, subjects were informed that they would be presented with a series of images of faces, butterflies, and moths, and common objects, and were not expected to remember or make judgments about the stimuli but to simply look at each image as it appeared. In Session 2, which occurred approximately $30 \mathrm{~min}$ after Session 1, subjects were instructed that they would see a further series of faces, butterflies, and moths, and objects, and would have to respond (as for the individuation condition above) if they were certain that the image was one they had been shown previously. (Note that all images had been seen previously but in different orders.)

For each viewing session, there were four runs, each lasting $1 \mathrm{~min} 48 \mathrm{sec}$. Each run consisted of four epochs of 18 images, one epoch of each stimulus category, fixation alone $(+)$, faces $(\mathrm{F})$, Lepidoptera (L), and objects (O) (see Figure 3). Images were presented for 750 msec with a 750-msec interstimulus interval consisting of a blank white screen (total of 27 sec/epoch). The order of epochs in the four runs was determined using a Latin square design in which each epoch type occurred in each position (first, second, third, or fourth) and each epoch type followed each other once. The run order across subjects was also determined using a Latin square design. 


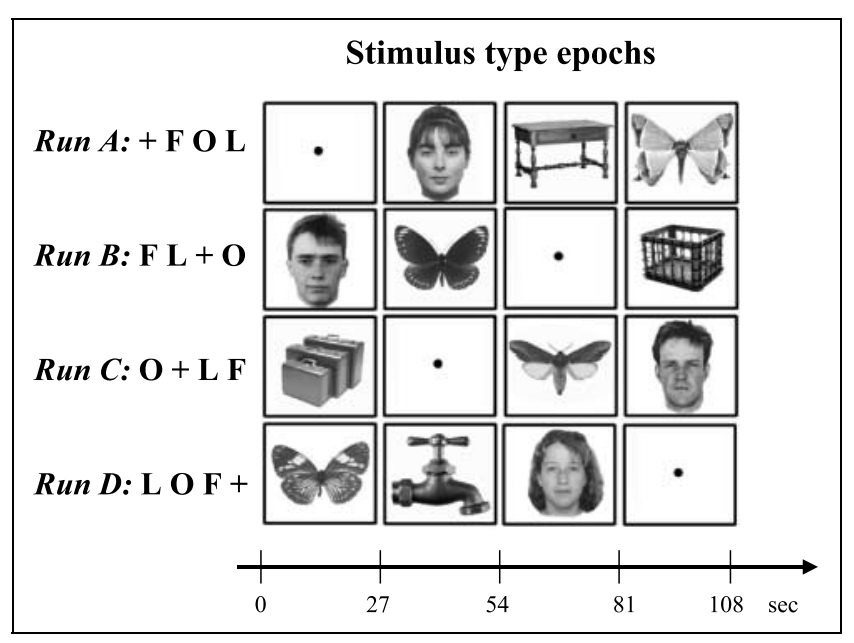

Figure 3. A schematic representation of the four runs of the main task of Experiments 1 and 2 showing examples of stimuli from each category. $\mathrm{F}=$ faces; $\mathrm{O}=$ objects; $\mathrm{L}=$ lepidoptera.

Sets of 18 images of each type were randomly assigned to runs for each subject/condition (except equal numbers of male/female faces and butterflies/moths per epoch). The TR of $2.1 \mathrm{sec}$ allowed 12.8 volumes per epoch.

\section{fMRI Acquisition and Analysis}

Functional images encompassing striate and extrastriate cortex $(18$ contiguous axial slices, in-plane resolution $=$ $4 \mathrm{~mm}$, slice thickness $=3 \mathrm{~mm}$, gap $=1 \mathrm{~mm}$ ) were acquired with a Siemens Vision (1.5 T) MRI system using a gradient-echo echoplanar imaging sequence ( $\mathrm{TR}=$ $2100 \mathrm{msec}, \mathrm{TE}=50 \mathrm{msec}$, flip $=90$, matrix $=64 \times 64$, FOV $=256 \times 256 \mathrm{~mm}$ ) from Deichmann and Turner (Wellcome Department of Cognitive Neurology, London, UK). A high-resolution anatomical image (MPRAGE, 170 contiguous sagittal slices, isovoxel $1 \mathrm{~mm}$ ) was also acquired for each subject.

The first three volumes from each functional run were discarded to ensure steady-state transverse magnetization. The functional images were realigned to correct for rigid body motion using SPM99 software (Wellcome Department of Cognitive Neurology). Global intensity normalization (mean 1000) and temporal smoothing (Gaussian filter, FWHM = 4 sec) were carried out using MEDX 3.3 (Sensor Systems).

All analyses incorporated a 4.2 -sec (2 volume) hemodynamic lag. Image analysis was performed using two different approaches. ROI-based analyses examined how the fMRI signal in the FFA, as defined functionally in a localizer task, changed as a function of viewing condition and stimulus category in the main task. FFA ROIs were first identified in each subject's left and right FGs by selecting all voxels showing significantly greater activation to faces than objects (KS test, $z>3.08, p<.001$, uncorrected) in the localizer task. Next, for each subject, the fMRI signal intensity time series during the main task (faces, objects, Lepidoptera, fixation) was extracted for each voxel in the previously functionally defined FFA ROIs. The mean percent signal change (relative to fixation) within each FFA ROI was calculated for each subject, viewing condition, and stimulus category.

In the regional overlap analyses, we determined the extent to which faces and Lepidoptera activated the same voxels within the FG, defined anatomically. First, the functional images were transformed to a standard stereotaxic space (Montreal Neurological Institute [MNI] template) using SPM99 (12-parameter affine transformation followed by a nonlinear warp using $7 \times$ $8 \times 7$ basis functions and bilinear interpolation). Left and right FG ROIs were then defined anatomically to incorporate all of the FG in accordance with atlas of Talairach and Tournoux (1988). Within each ROI for each subject, the following were determined: the number of voxels significantly more active $(p<.001$, uncorrected, $z>3.08$ ) to faces than objects, the number of voxels significantly more active to Lepidoptera than objects, and the number of voxels jointly activated in these two comparisons. The latter is the key measure for determining the extent of overlap in FG activation to faces and Lepidoptera.

\section{Experiment 2}

\section{Participants}

In Experiment 2, eight right-handed Lepidoptera experts (age 25-47 years, $M=37.3, S D=9.6,3$ women) were tested in the passive viewing condition. While they were older on average than the two groups of novice subjects of Experiment 1, the age difference was not significant, $F(2,23)=2.69, p<.09$.

The Lepidoptera experts completed a questionnaire detailing the number of years experience with Lepidoptera in general, experience with particular species, as well as formal qualifications in entomology. Additionally, they were asked to describe the form that their interest took (e.g., observation, photography, etc). Of the eight experts, four had completed formal study in aspects of entomology ranging from a full postgraduate degree in general entomology to being involved in specific research projects involving collection and dissection of various species of Lepidoptera. Their experience ranged from 6 to 30 years. The other four experts had been involved identifying Lepidoptera from observation, photography, or cataloguing specimens. Their experience ranged from 3 to 15 years. The overall mean years of experience was 12.8 years $(S D=$ 9.4 years).

An additional 11 nonexpert subjects (all students at University of Western Australia, age 17-34 years, $M=$ 20.9, $S D$ 5.6, 8 women) were recruited for a behavioral task to assess the degree of expertise of the Lepidoptera 
experts. The nonexperts were significantly younger than the experts, $F(1,15)=18.56, p<.001$.

\section{Procedure}

The imaging procedure (including stimuli and imaging sequences for both localizer and main tasks) was identical to that for the untrained novices of Experiment 1, with passive viewing in Session 1 and individuation in Session 2 . Only experts participated in the imaging sessions.

To assess the level of expertise of the experts, experts and nonexperts completed an old/new recognition task and a classification task. In the old/new task, subjects studied 36 Lepidoptera (18 butterflies, 18 moths, different from those seen in the scanner, but from the same source), displayed individually on cards, for $3 \mathrm{sec}$ each, in random order. Immediately following the study phase, subjects were given a two-alternative forcedchoice test consisting of 36 pairs of Lepidoptera, and asked to indicate which member of each pair was old. Following the old/new task, subjects completed a categorization task in which they sorted all 72 images into six families (three for butterflies, three for moths).

\section{Group Analysis}

To determine the locations of Lepidoptera and face activation, the data were spatially normalized to the MNI standard brain, then smoothed (6-mm isotropic FWHM Gaussian kernel). For individual subjects, condition effects (faces, Lepidoptera, and objects) were estimated according to the general linear model using SPM99. Stimulus-related activation was modeled as boxcar functions convolved with the hemodynamic response function. Linear contrasts were specified to investigate differential effects of Lepidoptera versus objects (LO) and faces versus objects (FO). Each contrast produced a map of the weighted parameter estimates suitable for input to a second-level (group) analyses. Group results were obtained using one sample $t$ tests performed on the individual subject contrast images of LO for untrained novices $(n=9)$, LO for experts $(n=8)$, and FO for experts $(n=8)$. Analyses were restricted to Session 1 (passive viewing). The resultant $t$ maps (thresholded at $p<.05$, uncorrected) are shown superimposed on the appropriate mean group anatomical image in Figure 2. The center of FO activation is indicated by the crosshairs in Figure 2. Its Talairach and Tournoux (1988) coordinates were 43.6, $-47.3,-14.4$ (calculated using the mni2tal algorithm developed by M. Brett, Cambridge, MA) (MNI coordinates: $44,-48,-20$ ).

\section{Acknowledgments}

This research was supported by a Large ARC Grant A10019011 to Puce, Rhodes, and Michie, and a Small ARC Grant
(University of Western Australia) to Rhodes and Michie. We thank Ian Morris and Allison Melsun of the MRI Unit, Sir Charles Gairdner Hospital, for assistance with scanning. We also thank Peter Clissa, Fiona Michel, Marianne Peters, and Linda Jeffreys for assistance with the imaging sessions, subject recruitment, and collection of behavioral data. We are indebted to N. Kanwisher for providing the face and object stimuli used in the face localizer task and to R. Deichmann and R. Turner for access to the echoplanar imaging sequence.

Reprint requests should be sent to Gillian Rhodes, School of Psychology, University of Western Australia, Crawley, Perth, Western Australia 6009, Australia, or via e-mail: gill@psy.uwa. edu.au.

The data reported in this experiment have been deposited in the fMRI Data Center (http://www.fmridc.org). The accession number is 2-2003-114BN.

\section{Notes}

1. It is important to note that faces can also activate objectsensitive regions (McCarthy et al., 1997; Malach et al., 1995), which may respond to a face as an object (McCarthy et al., 1997) and play a role in face detection.

2. Experts tend to spontaneously individuate objects with which they have expertise (Tanaka \& Taylor, 1991; Tanaka, 2001), so that individuation should be observed in a passive viewing task

3. The stimulus category main effect and Category $\times$ Group interaction were significant for a number of activation measures, including \%MR signal change relative to overall mean signal intensity and mean signal intensities within the FFA ROI (and with fixation as another level of the category factor) defined using either the selected criterion $(p<.001)$ or the more conservative criterion previously used by Kanwisher et al. (1997) to identify the FFA ROI. The Category $\times$ Hemisphere interaction was less robust across these different analyses and therefore will not be considered further. 4. Performance on the old/new judgments was unrelated to the extent of activation in either the left or right FFA.

\section{REFERENCES}

Allison, T., Ginter, H., McCarthy, G., Nobre, A. C., Puce, A., Luby, M., \& Spencer, D. D. (1994). Face recognition in the human extrastriate cortex. Journal of Neurophysiology, 71, 821-825.

Allison, T., McCarthy, G., Nobre, A., Puce, A., \& Belger, A. (1994). Human extrastriate visual cortex and the perception of faces, words, numbers and colors. Cerebral Cortex, 5, $544-554$.

Allison, T., Puce, A., Spencer, D. D., \& McCarthy, G. (1999). I. Potentials generated in occipitotemporal cortex by face and non-face stimuli. Cerebral Cortex, 9, 415-430.

Bahrick, H. P, Bahrick, P. O., \& Wittlinger, R. P. (1975). Fifty years of memory for names and faces: A cross-sectional approach. Journal of Experimental Psychology: General, 104, 54-75.

Bartlett, J. C., \& Searcy, J. (1993). Inversion and configuration of faces. Cognitive Psychology, 25, 281-316.

Biederman, I. (1987). Recognition-by-components: A theory of human image understanding. Psychological Review, 94, 115-147.

Bushnell, I. W. R. (2001). Mother's face recognition in newborn infants: Learning and memory. Infant and Child Development, 10, 67-74.

Damasio, A. R., Damasio, H., \& Van Hoesen, G. W. (1982). 
Prosopagnosia: Anatomic basis and behavioral mechanisms. Neurology, 32, 331-341.

De Renzi, E., Faglioni, P., Grossi, D., \& Nichelli, P. (1991). Apperceptive and associative forms of prosopagnosia. Cortex, 27, 213-221.

De Renzi, E., Perani, D., Carlesimo, G. A., Silveri, M. C., \& Fazio, F. (1994). Prosopagnosia can be associated with damage confined to the right hemisphere-An MRI and PET study and a review of the literature. Neuropsychologia, 32, 893-902.

Desimone, R. (1991). Face-selective cells in the temporal cortex of monkeys. Journal of Cognitive Neuroscience, 3, 1-8.

Diamond, R., \& Carey, S. (1986). Why faces are and are not special: An effect of expertise. Journal of Experimental Psychology: General, 115, 107-117.

Farah, M. J. (1996). Is face recognition "special"? Evidence from neuropsychology. Behavioral Brain Research, 76, 181-189.

Farah, M. J., Wilson, K. D., Drain, H. M., \& Tanaka, J. R. (1995). The inverted face inversion effect in prosopagnosia: Evidence for mandatory, face-specific perceptual mechanisms. Vision Research, 35, 2089-2093.

Fodor, J. (1983). Modularity of mind. Cambridge: MIT Press.

Gauthier, I., Anderson, A. W., Tarr, M. J., Skudlarski, P., \& Gore, J. C. (1997). Levels of categorization in visual recognition studied using functional magnetic resonance imaging. Current Biology, 7, 645-651.

Gauthier, I., Skudlarski, P., Gore, J. C., \& Anderson, A. W. (2000). Expertise for cars and birds recruits brain areas involved in face recognition. Nature Neuroscience, 3, 191-197.

Gauthier, I., \& Tarr, M. J. (1997). Becoming a "greeble" expert: Exploring the face recognition mechanisms. Vision Research, 37, 1673-1682.

Gauthier, I., Tarr, M. J., Anderson, A. W., Skudlarski, P., \& Gore, J. C. (1999). Activation of the middle fusiform "face area" increases with expertise in recognizing novel objects. Nature Neuroscience, 2, 568-573.

Gauthier, I., Tarr, M. J., Moylan, J., Anderson, A. W., Skudlarski, P., \& Gore, J. C. (2000). Does visual subordinate-level categorisation engage the functionally defined fusiform face area? Cognitive Neuropsychology, 17, 143-163.

Gauthier, I., Tarr, M. J., Moylan, J., Skudlarski, P., Gore, J. C., \& Anderson, A. W. (2000). The fusiform "face area" is part of a network that processes faces at the individual level. Journal of Cognitive Neuroscience, 12, 495-504.

George, N., Dolan, R. J., Fink, G. R., Baylis, G. C., Russell, C., \& Driver, J. (1999). Contrast polarity and human face recognition in the human fusiform gyrus. Nature Neuroscience, 2, 574-580.

Golby, A. J., Gabrielle, J. D. E., Chiao, J. Y., \& Eberhardt, J. L. (2001). Differential responses in the fusiform region to same-race and other-race faces. Nature Neuroscience, 4, 845-850.

Harries, M. H., \& Perrett, D. I. (1991). Visual processing of faces in temporal cortex: Physiological evidence for a modular organization and possible anatomical correlates. Journal of Cognitive Neuroscience, 3, 9-24.

Haxby, J. V., Gobbini, M. I., Furey, M. L., Ishai, A., Schouten, J. L., \& Piertrini, P. (2001). Distributed and overlapping representations of faces and objects in ventral temporal cortex. Science, 293, 2425-2430.

Haxby, J, V., Hoffman, E. A., \& Gobbini, M. I. (2000). The distributed human neural system for face perception. Trends in Cognitive Science, 4, 223-233.

Haxby, J. V., Ungerleider, L. G., Clark, V. P., Schouten, J. L., Hoffman, E. A., \& Martin, A. (1999). The effect of face inversion on activity in human neural systems for face and object perception. Neuron, 22, 189-199.

Johnson, M. H., Dziurawiec, S., Ellis, H., \& Morton, J. (1991). Newborns' preferential tracking of face-like stimuli and its subsequent decline. Cognition, 40, 1-19.

Kanwisher, N. (2000). Domain specificity in face perception. Nature Neuroscience, 3, 759-763.

Kanwisher, N., Chun, M. M., McDermott, J., \& Hamilton, R. (1996). fMRI reveals distinct extrastriate loci selective for faces and objects. Society of Neuroscience Abstracts, 22, 1937.

Kanwisher, N., McDermott, J., \& Chun, M. M. (1997). The fusiform face area: A module in human extrastriate cortex specialized for face perception. Journal of Neuroscience, 17, 4302-4311.

Le Grand, R., Mondloch, C. J., Maurer, D., \& Brent, H. P. (2001). Early visual experience and face processing. Nature, 410, 890

Leopold, D. A., O’ Toole, A. J., Vetter, T., \& Blanz, V. (2001). Prototype-referenced shape encoding revealed by high-level after effects. Nature Neuroscience, 4, 89-94.

Logothetis, N. K., Pauls, J., \& Poggia, T. (1995). Shape representation in the inferior temporal cortex of monkeys. Current Biology, 5, 552-563.

Logothetis, N. K. (1998, June). Single neurons and visual perception. Paper presented at the International Neuropsychological Symposium, Israel.

Malach, R., Reppas, J. B., Benson, R. R., Kwong, K. K., Jiang, H., Kennedy, W. A., Ledden, P. J., Brady, T. J., Rosen, B. R., \& Tootell, R. B. H. (1995). Object-related activity revealed by functional magnetic resonance imaging in human occipital cortex. Proceedings of the National Academy of Sciences, U.S.A., 92, 8135-8139.

McCarthy, G., Puce, A., Gore, J. C., \& Allison, T. (1997). Face-specific processing in the human fusiform gyrus. Journal of Cognitive Neuroscience, 9, 604-609.

McCarthy, R., \& Warrington, E. K. (1986). Visual associative agnosia: A clinico-anatomical study of a single case. Journal of Neurology, Neurosurgery and Psychiatry, 49, 1233-1240.

Moscovitch, M., Winocur, G., \& Behrmann, M. (1997). What is special about face recognition: Nineteen experiments on a person with visual object agnosia and dyslexia but normal face recognition. Journal of Cognitive Neuroscience, 9, 555-604.

Murray, J. E., Yong, E., \& Rhodes, G. (2000). Revisiting the perception of upside-down faces. Psychological Science, 11, 492-496.

Oldfield, R. C. (1971). The assessment and analysis of handedness: The Edinburgh Inventory. Neuropsychologia, 9, 97-113.

Peterson, M. P., \& Rhodes, G. (2003). Perception of faces, objects and scenes: Analytic and holistic processing. Cambridge, MA: Oxford University Press.

Price, C. J., Moore, C. J., Humphreys, G. W., Frackowiak, R. S., \& Friston, K. J. (1996). The neural regions sustaining object recognition and naming. Proceedings of the Royal Society of London Series B: Biological Science, 263, 1501-1507.

Puce, A., Allison, T., Asgari, M., Gore, J. C., \& McCarthy, G. (1996). Differential sensitivity of human visual cortex to faces, letterstrings, and textures: A functional MRI study. Journal of Neuroscience, 16, 5205-5215.

Puce, A., Allison, T., Gore, J. C., \& McCarthy, G. (1995). A functional MRI study of face perception in extrastriate cortex. Journal of Neurophysiology, 74, 1192-1199.

Puce, A., Allison, T., \& McCarthy, G. (1999). Electrophysiological studies of human face perception: III. 
Effects of top-down processing on face-specific potentials. Cerebral Cortex, 9, 445-458.

Rhodes, G. (1996). Superportraits: Caricatures and recognition. Hove: Psychology Press.

Rhodes, G., Brake, S., \& Atkinson, A. P. (1993). What's lost in inverted faces. Cognition, 47, 25-57.

Rosch, E., Mervis, C. B., Gray, W. D., Johnson, D. M., \& Boyes-Braem, P. (1976). Basic objects in natural categories. Cognitive Psychology, 8, 382-439.

Rossion, B., Dricot, L., Devolder, A., Bodart, J.-M., \& Crommelinck, M. (2000). Hemispheric asymmetries for whole-based and part-based face processing in the human fusiform gyrus. Journal of Cognitive Neuroscience, 12, 793-802.

Searcy, J. H., \& Bartlett, J. C. (1996). Inversion and processing of component and spatial-relational information in faces. Journal of Experimental Psychology: Human Perception and Performance, 22, 904-915.

Sergent, J., Ohta, S., \& MacDonald, B. (1992). Functional neuroanatomy of face and object processing: A positron emission tomography study. Brain, 115, 15-36.
Talairach, J., \& Tournoux, P. (1988). Co-planar stereotaxic atlas of the human brain. New York: Thieme.

Tanaka, J. W. (2001). The entry point of face recognition: Evidence for face expertise. Journal of Experimental Psychology: General, 130, 534-543.

Tanaka, J. W., \& Taylor, M. (1991). Object categories and expertise: Is the basic level in the eye of the beholder? Cognitive Psychology, 23, 457-482.

Tarr, M. J., \& Cheng, Y. D. (2003). Learning to see faces and objects. Trends in Cognitive Sciences, 7, 23-30.

Tarr, M. J., \& Gauthier, I. (2000). FFA: A flexible fusiform area for subordinate-level visual processing automatized by expertise. Nature Neuroscience, 3, 764-769.

Walton, G. E., \& Bower, T. G. R. (1993). Newborns form "prototypes" in less than one minute. Psychological Science, 4, 203-205.

Wojciulik, E., Kanwisher, N., \& Driver, J. (1998). Covert visual attention modulates face-specific activity in human fusiform gyrus: fMRI study. Journal of Neurophysiology, 79, 1574-1578. 